Discussion This is the only case that has ever been observed in the careers of our staff of at our high-volume aneurysm center. The likelihood of this occurrence is certainly extraordinarily small. Nevertheless, high clinical suspicion is paramount for a fast, stepwise, and effective therapeutic response. The patient was unsupervised and it was only by chance that her deterioration was necessarily witnessed. In collaboration with out CT technologists, we held instituted in-services during which our staff were educated about ominous imaging findings and encouraged to emergently call a radiologist to confirm these. We propose that centers performing neuroimaging should at the very least be prepared to both supervise and initially manage patients who deteriorate. In addition, while many radiographers will inform an attending radiologist or another physician when an imaging finding appears concerning, standardization in the recognition and reporting of some acute pathological entities may improve detection and response in imaging departments.

Disclosures A. Dmytriw: None. J. Martinez Santos: None. J. Spears: None. T. Marotta: None.

\section{E-063 PERCUTANEOUS BLEOMYCIN SCLEROTHERAPY FOR CONJUNCTIVAL VENOUS AND LYMPHATIC MALFORMATIONS}

A Berenstein. Neurosurgery, Mount Sinai Health System, New York, NY

\subsection{6/neurintsurg-2016-012589.135}

Purpose To describe the use of bleomycin in a foam preparation and or undiluted in slow flow vascular lesions that involve the conjunctiva as the sole treatment

Material and methods 5 patients with venous malformations, and 2 with lymphatic malformations involving the conjunctiva were treated with various combination bleomycin injections in to the lesion under direct puncture, using various needles and or angio-catheters; and monitored with US or DSA.

Results There was significant improvement, or near total resolution in all patients with a follow up of up to 3 years, there were no complications

Conclusions The use of bleomycin in various forms appears as a simple, safe and very effective treatment for low flow vascular lesions involving the conjunctiva, avoiding more elaborated and challenging surgical intervention.

Disclosures A. Berenstein: None.

\section{E-064 ADJUNCTIVE STENT ARE NOT FLOW DIVERTERS: A COMPUTATIONAL FLOW DYNAMICS STUDY COMPARING FLOW DIVERTERS TO ADJUNCTIVE STENTS}

${ }^{1} \mathrm{~B}$ Jankowitz, ${ }^{2} \mathrm{~F}$ lannaccone, ${ }^{3} \mathrm{G}$ De Santis, ${ }^{3} \mathrm{M}$ De Beule, ${ }^{4} \mathrm{M}$ Gounis, ${ }^{5} \mathrm{~K}$ Van Der Marel, ${ }^{5}$ A Puri. ' Neurosurgery, UPMC, Pittsburgh, PA; ${ }^{2}$ Neurosurgery, UPMC, Pittsburgh, Belgium; ${ }^{3}$ FEops nv, Gent, Belgium; ${ }^{4}$ Radiology, UMASS, Worcester, MA; ${ }^{5}$ Neurosurgery, UMASS, Worcester, MA

\subsection{6/neurintsurg-2016-012589.136}

Introduction Historically, adjunctive stents had different constructions than flow diverters. The former were open- (e.g. Neuroform) or closed-cell (e.g. Enterprise) laser cut nitinol devices, with low metal-to-artery ratios and pore densities. The recent introduction of woven adjunctive stents (e.g. LVIS
Jr., Leo Baby) blurred the line between adjunctive stents and flow diverters (e.g. Pipeline Embolization Device or PED). There is a misconception that woven adjunctive stents have flow diverting capabilities. A computational flow dynamic (CFD) model was used to compare the flow alteration effects of two adjunctive stents (Neuroform Atlas and LVIS Jr.) and one flow diverter (PED).

Materials and methods A CFD study was performed on a 2.6 $\mathrm{mm}$ sidewall aneurysm in a $2.2-2.3 \mathrm{~mm}$ diameter vessel. Three stents were modeled: Neuroform Atlas $(3.0 \times 24 \mathrm{~mm})$, LVIS Jr. $(3.5 \times 20 \mathrm{~mm})$, PED $(2.75 \times 10 \mathrm{~mm})$. The stent was virtually deployed in a compliant vessel model with a wall thickness of $0.6 \mathrm{~mm}$ using Finite Element Analysis. For the flow conditions, stress free outlet was assumed with a steady state flow of $2.1 \mathrm{~mL} / \mathrm{s}$. Six CFD runs were performed on each adjunctive stent with varying degrees of rotation and placement. The following definitions were used; \% aneurysm inflow $=$ inflow rate/parent artery flow rate, turnover time $=$ aneurysm volume/inflow rate, impact zone $=$ area of aneurysm with WSS $>2 \mathrm{~Pa}$. Two-sample T-tests compared Atlas to LVIS Jr.

Results The CFD study revealed that the 2 adjunctive stents did not divert a significant amount of flow when compared to a flow diverter (Table 1). When compared to each other, the average values for $\%$ aneurysm inflow $(\mathrm{P}=0.265)$, turnover time $(P=0.960)$, and impact zone $(P=0.135)$ were not statistically different. In contrast, the PED significantly reduced \% aneurysm inflow, turnover time, and impact zone. Conclusions This analysis revealed that adjunctive stents do not divert flow significantly when compared to pre-treatment baselines. Only a flow diverter significantly altered the flow dynamics within the aneurysm. There was no statistically significant difference in the flow diversion capabilities between the two adjunctive stents.
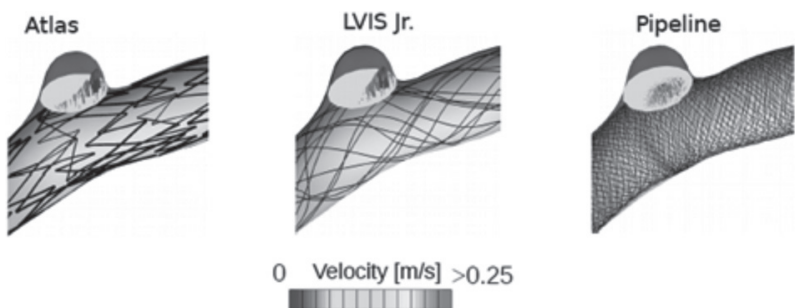

Velocity components perpendicular to the neck plane

Abstract E-064 Figure 1

Abstract E-064 Table 1 Averaged results from CFD analysis of 2 adjunctive stents and a PED

\begin{tabular}{llll}
\hline Model & $\%$ Aneurysm inflow & Turnover time $(\mathrm{S})$ & Impact zone $\left(\mathrm{mm}^{2}-\%\right)$ \\
\hline Pre-treatment & $8 \%$ & 0.0154 & $7.4-98 \%$ \\
Atlas & $6.2 \%$ & 0.0215 & $7.43-94 \%$ \\
LVIS Jr. & $5.8 \%$ & 0.0213 & $6.8-89 \%$ \\
PED & $1.4 \%$ & 0.09 & $0.04-0.6 \%$ \\
\hline
\end{tabular}

Disclosures B. Jankowitz: None. F. Iannaccone: None. G. De Santis: None. M. De Beule: None. M. Gounis: None. K. Van Der Marel: None. A. Puri: None. 